\title{
Adaptive Fuzzy Sliding Mode Control for Uncertain Nonlinear Systems
}

\author{
Samjun Seo \\ Dept. of Electrical and Electronic Engineering, Anyang University \\ 708-113 Anyang 5-dong, Manan-gu Anayng-shi Gyeonggi-do 430-7143, Korea
}

\begin{abstract}
This paper deals with a new adaptive fuzzy sliding mode controller and its application to an inverted pendulum. We propose a new method of adaptive fuzzy sliding mode control scheme that the fuzzy logic system is used to approximate the unknown system functions in designing the SMC of uncertain nonlinear systems. The controller's construction and its analysis involve sliding modes. The proposed controller consists of two components. Sliding mode component is employed to eliminate the effects of disturbances, while a fuzzy model component equipped with an adaptation mechanism reduces modeling uncertainties by approximating model uncertainties. To demonstrate its performance, the proposed control algorithm is applied to an inverted pendulum. The results show that both alleviation of chattering and performance are achieved.
\end{abstract}

Key words : fuzzy basis function, uncertain nonlinear system, fuzzy sliding mode control, inverted pendulum

\section{Introduction}

Variable structure control systems constitute a class of nonlinear feedback control systems whose structure changes depending on the state of the system. Due to its good robustness to uncertainties, sliding mode control has been accepted as an efficient method for robust control of uncertain systems. Being limited only by practical constraints on the magnitude of control signal, the sliding mode controller can treat a variety of uncertainties as well as bounded external disturbances. A key step in the design of controllers is to introduce a proper transformation of tracking errors to generalized errors so that an nth-order tracking problem can be transformed into an equivalent first-order stabilization problem. Since the equivalent first-order problem is likely to be simple to handle, a control law may be easily developed to achieve the so-called reaching condition [1].

Despite the benefits of VSC control, this suffers from two major shortcomings. First, the insensitivity property of a VSC system is present only when the system is in the sliding mode. The second shortcoming is control chattering. To overcome the first disadvantage, that is to reduce the reaching time, the use of high gain control signal was suggested [2]. On the other hand, a time-varing switching surface was suggested in order to eliminate the reaching phase, where the initial tracking control errors were assumed to be zero [3]. However, this assumption rules out many practical situations in which the system initial conditions are located arbitrarily.

The problem for chattering has been addressed by many researchers. In [3], the discontinuous control is approximated inside a boundary layer located around the switching surface. However, although chattering can be made negligible if the

Manuscript received Nov. 9, 2010; revised Mar. 2, 2011; Accepted Mar. 10, 2011. width of the boundary layer is chosen large enough, the guaranteed tracking precision will deteriorate if the available control bandwidth is limited.

The fuzzy logic controller, based on Zadeh' s fuzzy set theory has firstly been developed by Mamdani and his coworkers about twenty years ago, and has successfully been applied to many commercial products and industrial systems [4][5]. The main advantages of fuzzy logic controller are it can control the complex ill-defined systems by converting the linguistic control strategy of operators' experience or experts' knowledge into an automatic control strategy without knowing the mathematical model of the controlled systems.

However, at present there still have some problems in the design of fuzzy logic controllers. Control rules that are the most important factor in FLC are generally obtained from intuition and experience of the experts, and such rules represented by linguistic rule sets or fuzzy relation. But there is always something difficult to obtain from such control rules and this makes the design of the controller difficult and the response trajectory of the controlled system is unpredictable.

Recently, fuzzy logic control has emerged as a paradigm of intelligent control capable of dealing with complex and illdefined systems [6]. New results have been made recently to identify the connection between fuzzy logic and variable structure control $[7,8,9]$. It has been shown that fuzzy logic control is a general form of variable structure control. This connection has suggested the integration of the two control approaches in control system design applications $[8,9,10]$.

In this paper, a new approach to the design of an adaptive fuzzy sliding mode controller is proposed. By defining a new complementary sliding variable to the conventional sliding variable, a novel fuzzy logic system is used to approximate nthorder nonlinear systems. We will provide a new theoretical framework for the construction of an adaptive fuzzy sliding 
mode controller. The proposed controller will be shown to result in closed-loop system satisfying a general sliding mode condition such that a region containing the hyperplane, defined by the two sliding surfaces, is attractive and positively invariant. In addition, we will show that this control scheme will reduce the ultimate bound tracking error at least by one-half, as compared to the conventional sliding mode control. More importantly, it will be shown to significantly improve the tracking performance during the reaching phase as well as in the sliding mode.

In design of FLC, fuzzy control rules are obtained from expert's experience and intuition and it is very difficult to obtain them. We proposed an adaptive algorithm which is automatically updated by consequence parameters of control rules in order to reduce chattering phenomenon and simultaneously to satisfy the sliding mode condition. To demonstrate its performance, the proposed control algorithm is applied to an inverted pendulum system. The results show that both alleviation of chattering and performance are achieved.

\section{Adaptive Fuzzy Systems}

\subsection{Fuzzy model identification}

A fuzzy system consists of three parts: fuzzifier, fuzzy rules and defuzzifier.

The fuzzifier maps an input point in the input space $U \in R^{n}$ to a fuzzy set $A_{x}$ in the space. Fuzzy sets are characterized by their membership functions $\mu(\cdot) \mathrm{s}$. The inference engine performs a mapping from fuzzy sets in the input space to fuzzy sets in the output space $V \in R^{m}$ based on fuzzy rules

In this paper, we consider the case where the fuzzy rule base consists of $N=\prod_{j=1}^{n} N_{j}$ rules for a multi-input and singleoutput (MISO) in the following form:

$$
\begin{gathered}
R_{i_{1}, i_{2}, \cdots, i_{n}} \text { : IF } x_{1} \text { is } A_{i_{i}}^{1} \text { and } x_{2} \text { is } A_{i_{2}}^{2} \text { and } \cdots \text { and } x_{n} \text { is } A_{i_{n}}^{n} \\
\text { THEN } y \text { is } C_{i_{1}, i_{2}, \cdots, i_{n}}
\end{gathered}
$$

with $i_{1}=1,2, \ldots, N_{1}, \quad i_{2}=1,2, \ldots, N_{2}, \ldots, \quad i_{n}=1,2, \ldots, N_{n}, \quad x_{j}$, $j=1,2, \ldots, n$ are the input variables of the fuzzy system, $y$ is the output variable of the fuzzy system, and fuzzy set $A_{i_{j}}^{j} \in U_{j}$ and $C_{i_{1}, i_{2}, \cdots, i_{n}} \in V$ are linguistic terms characterized by fuzzy membership functions $A_{i_{j}}^{j}\left(x_{j}\right)$ and $C_{i_{1}, i_{2}, \cdots, i_{n}}(y)$, respectively. By using the singleton fuzzifier, product inference engine, and the center-average defuzzifier, the set of fuzzy systems can be expressed as follows:

$$
\begin{aligned}
& y=\frac{\sum_{i_{1}, i_{2}, \cdots, i_{n} \in I} A_{i_{1}, i_{2}, \cdots, i_{n}}(\mathbf{x}) y_{i_{1}, i_{2}, \cdots, i_{n}}}{\sum_{i_{1}, i_{2}, \cdots, i_{n} \in I} A_{i_{1}, i_{2}, \cdots, i_{n}}(\mathbf{x})} \\
& =\sum_{i_{1}, i_{2}, \cdots, i_{n} \in I}\left[\frac{\prod_{j=1}^{n} A_{i_{j}}^{j}\left(x_{j}\right)}{\sum_{i_{1}, i_{2}, \cdots, i_{n} \in I} \prod_{j=1}^{n} A_{i_{j}}^{j}\left(x_{j}\right)}\right] y_{i_{1}, i_{2}, \cdots, i_{n}}
\end{aligned}
$$

Where $\quad \mathbf{x}=\left[x_{1}, x_{2}, \cdots, x_{n}\right]^{T} \in U_{1} \times U_{2} \times \cdots \times U_{n}=U \in R^{n}$ is state vectors and $y_{i_{1}, i_{2}, \cdots, i_{n}}$ is the point in $V$ at which $C_{i_{1}, i_{2}, \cdots, i_{n}}(y)$ achieves its maximum value and $A_{i_{1}, i_{2}, \cdots, i_{n}}(\mathbf{x})=\prod_{j=1}^{n} A_{i_{j}}^{j}\left(x_{j}\right)$. Without loss of generality, we assume that $C_{i_{1}, i_{2}, \cdots, i_{n}}\left(y_{i_{1}, i_{2}, \cdots, i_{n}}\right)$ is 1.

From (2) of the fuzzy system, it is easy to fine that the fuzzy system in fact can be represented by a linear combination of the functions

$$
\begin{aligned}
& \xi_{i_{1}, i_{2}, \cdots, i_{n}}=\frac{\prod_{j=1}^{n} A_{i}^{j}\left(x_{j}\right)}{\sum_{i_{1}, i_{2}, \cdots, i_{n} \in I} \prod_{j=1}^{n} A_{i_{j}}^{j}\left(x_{j}\right)}, \\
& i_{j}=1,2, \ldots, N_{j} \quad j=1,2, \ldots, n
\end{aligned}
$$

These functions can be defined as the fuzzy basis functions (FBFs) of the fuzzy system. Now the fuzzy system of (2) is equivalent to the FBF expansion

$$
y=\sum_{i_{1}, i_{2}, \cdots, i_{n} \in I} \xi_{i_{1}, i_{2}, \cdots, i_{n}}(\mathbf{x}) y_{i_{1}, i_{2}, \cdots, i_{n}}
$$

In order to develop learning algorithms for these fuzzy systems, we need to specify the functional form of fuzzy membership function for a fuzzy set $A_{i_{j}^{j}}^{j}\left(x_{j}\right)$. The membership function used in this paper is a Gaussian-shaped form

$$
A_{i_{j}}^{j}\left(x_{j}, p_{i j}, q_{i j}\right)=\exp \left[-\frac{\left(x_{j}-p_{i_{j}}\right)^{2}}{2 q_{i_{j}}^{2}}\right]
$$

with the centroid $p_{i j}$ and the width $q_{i j}$. If we view all the parameters $p_{i_{j}}$ and $q_{i j}$ in (5) as free design parameters, then the FBF expansion (4) is nonlinear in the parameters. On the other hand, we can fix all the parameters in $A_{i j}^{j}\left(x_{j}, p_{i j}, q_{i j}\right)$ at the very beginning of the FBF expansion design procedure, so that the only free design parameters are $y_{i_{1}, i_{2}, \cdots, i_{n}}$; in this case $y$ of (4) is linear in the parameters. The advantage of being linear-in-the parameters is that we are able to use some very efficient linear parameter estimation methods. If we view the $y_{i_{1}, i_{2}, \cdots, i_{n}}$ as adjustable parameters, then (4) can be written as

$$
y=\theta^{T} \xi(\mathbf{x})
$$

where $\theta$ is the collection of $y_{i_{1}, i_{2}, \cdots, i_{n}}$ and $\xi(\mathbf{x})$ is a collection of $\xi_{i_{1}, i_{2}, \cdots, i_{n}}(\mathbf{x})$. The fuzzy system in the form of (6) with (5) is the most frequently used one in control application.

\subsection{Adaptive fuzzy controller design}

In this section, we first set up the control objectives and then show how to design an adaptive controller based on the fuzzy system to achieve these control objectives.

Consider the nth-order nonlinear system of the form

$$
\begin{aligned}
& x^{(n)}=f\left(x, \dot{x}, \cdots, x^{(n-1)}\right)+g\left(x, \dot{x}, \cdots, x^{(n-1)}\right) u \\
& y=x
\end{aligned}
$$

where $f$ and $g$ are unknown continuous smooth nonlinear functions, $u \in R$ and $y \in R$ are input and output of the system, respectively, and $x \in R^{n}$ is the state vector of the system that is 
assumed to be available for measurement.

The control objective is to determine a feedback control input $u$ in order to force the output $y$ to track a given bounded reference signal $y_{m}$ and an adaptation law for adjusting the parameter vector $\theta$, under the constraint that all signals involved must be bounded.

In many practical applications, the control design has rough estimates of any unknown nonlinearities. To incorporate any such a priori information into our control design, the unknown functions $f$ and $g$ are expressed as

$$
\begin{aligned}
& f(\mathbf{x})=f_{n}(\mathbf{x})+\phi_{f}(\mathbf{x}) \\
& g(\mathbf{x})=g_{n}(\mathbf{x})+\phi_{g}(\mathbf{x})
\end{aligned}
$$

where $f_{n}$ and $g_{n}$ are known functions (representing some nominal estimates of $f$ and $g$, respectively) and $\phi_{f}$ and $\phi_{g}$ are unknown functions representing the system uncertainty.

Using this form allows the fuzzy systems to approximate the uncertainties $\phi_{f}$ and $\phi_{g}$ (whose magnitudes are often small) rather than the overall functions $f$ and $g$, respectively. An advantage of the above formulation is derived from the fact that if we turn off adaptation of the fuzzy system then we have a nominal for a nominal model, then $f_{n}$ and $g_{n}$ may be simply set to zero.

The unknown functions $\phi_{f}$ and $\phi_{g}$, representing the system uncertainties, are approximated by the fuzzy systems with rules of (1) in the form of (13) as follows;

$$
\begin{aligned}
& \hat{\phi}_{f}\left(\mathbf{x}, \theta_{f}\right)=\theta_{f}^{T} \xi_{f}(\mathbf{x}) \\
& \hat{\phi}_{g}\left(\mathbf{x}, \theta_{g}\right)=\theta_{g}^{T} \xi_{g}(\mathbf{x})
\end{aligned}
$$

where $\theta_{f}$ and $\theta_{g}$ are adjustable parameter vectors with appropriate dimensions, and $\xi_{f}(\mathbf{x}), \xi_{g}(\mathbf{x})$ are regressive vectors whose elements are fuzzy basis functions with the form of (3).

The uncertainties $\phi_{f}$ and $\phi_{g}$ can be expressed as

$$
\begin{aligned}
& \phi_{f}(\mathbf{x})=\hat{\phi}_{f}\left(\mathbf{x}, \theta_{f}^{*}\right)+\delta_{f}(\mathbf{x}) \\
& \phi_{g}(\mathbf{x})=\hat{\phi}_{g}\left(\mathbf{x}, \theta_{g}^{*}\right)+\delta_{g}(\mathbf{x})
\end{aligned}
$$

where $\theta_{f}^{*}$ and $\theta_{g}^{*}$ are some unknown optimal parameter vectors, and $\delta_{f}$ and $\delta_{g}$ represent the reconstruction errors for each fuzzy system. In general, increasing the number of the rules of the fuzzy systems reduces the reconstruction errors.

The optimal parameter vectors $\theta_{f}^{*}, \theta_{g}^{*}$ are artificial quantities required only for analytical purposes. Typically, $\theta_{f}^{*}$ and $\theta_{g}^{*}$ are chosen as the values of $\theta_{f}$ and $\theta_{g}$, respectively that minimize the reconstruction errors, i.e. ,

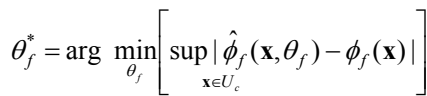

$$
\theta_{g}^{*}=\arg \min _{\theta_{g}}\left[\sup _{\mathbf{x} \in U_{c}} \hat{\phi}_{g}\left(\mathbf{x}, \theta_{g}\right)-\phi_{g}(\mathbf{x}) \mid\right]
$$

\section{Adaptive Fuzzy Sliding Mode Control}

\subsection{A new fuzzy sliding mode control scheme}

In this section, we will present a new sliding mode control scheme to serve as a theoretical basis on which development of a fuzzy sliding mode control can rest. The object is to find a continuous sliding control $u$ such that the output $y$ of (7) will approximately track a desired signal, $y_{d}$, which is assumed to be nth-order continuously differentiable and all of its derivates are uniformly bounded. Given the tracking

$$
e(t):=y(t)-y_{d}(t)
$$

Define the generalized error

$$
s(t)=\left(\frac{d}{d t}+\lambda\right)^{n-1} e(t)=\mathbf{c}^{T} \mathbf{e}(t)
$$

where $\quad \mathbf{e}(t)=\left[e_{1}, e_{2}, \ldots, e_{n}\right]^{T}=\left[\varepsilon, \dot{\varepsilon}, \ldots, \varepsilon^{(n-1)}\right]^{T} \quad, \quad \lambda>0 \quad, \quad$ and $\mathbf{c}^{T}=\left[c_{1}, c_{2}, \ldots, c_{n-1}, 1\right], \quad c_{j} \in R, \quad 1 \leq j \leq n-1$, are determined once the parameter $\lambda$ has been specified. The general error (17) provides a useful transformation such that the original nthorder tracking problem can be transformed into an equivalent 1st-order stabilization problem $s$ variable. Obviously, on the sliding surface, $s=0$, the tracking error , $e(t) \rightarrow 0$ as $t \rightarrow \infty$.

Differentiation of $s$ with respect to $t$, yields

$$
\begin{aligned}
\dot{s} & =\mathbf{c}^{T} \dot{\mathbf{e}}=\mathbf{c}^{T} \mathbf{A}_{c} \mathbf{e}+\mathbf{c}^{T} \mathbf{b}_{\mathbf{c}}\left(f(\mathbf{x})+g(\mathbf{x}) u-y_{d}^{(n)}\right) \\
& =\overline{\mathbf{c}}^{T} \mathbf{e}+\left(f(\mathbf{x})+g(\mathbf{x}) u-y_{d}^{(n)}\right) \\
& =f(\mathbf{x})-w+g(\mathbf{x}) u
\end{aligned}
$$

where $\overline{\mathbf{c}}^{T}=\left[0, c_{1}, c_{2}, \ldots, c_{n-1}\right], \quad w=\overline{\mathbf{c}}^{T} \mathbf{e}+y_{d}^{(n)}$, and

$$
\mathbf{A}_{\mathbf{c}}=\left[\begin{array}{ccccc}
0 & 1 & 0 & \cdots & 0 \\
0 & 0 & 1 & \cdots & 0 \\
\vdots & \vdots & \vdots & \ddots & \vdots \\
0 & 0 & 0 & \cdots & 0 \\
0 & 0 & 0 & \cdots & 0
\end{array}\right], \quad \mathbf{b}_{\mathbf{c}}=\left[\begin{array}{c}
0 \\
0 \\
\vdots \\
0 \\
1
\end{array}\right], \quad \mathbf{c}_{c}=\left[\begin{array}{llll}
1 & 0 & \cdots & 0
\end{array}\right] .
$$

If $f$ and $g$ were known, the equivalent control $u_{e q}=-\frac{1}{g(\mathbf{x})}[f(\mathbf{x})-w]$ would render $\dot{s}=0$. However, $f$ and $g$ are unknown, fuzzy approximators $\hat{f}, \hat{g}$, for $f$ and $g$, respectively, should be employed and certainty equivalent control law would be of the form

$$
\hat{u}_{e q}=-\frac{1}{\hat{g}(\mathbf{x})}[\hat{f}(\mathbf{x})-w]
$$

where

$$
\hat{f}(\mathbf{x})=f_{n}(\mathbf{x})+\hat{\phi}_{f}(\mathbf{x})=f_{n}(\mathbf{x})+\theta_{f}^{T} \xi_{f}(\mathbf{x})
$$




$$
\hat{g}(\mathbf{x})=g_{n}(\mathbf{x})+\hat{\phi}_{g}(\mathbf{x})=g_{n}(\mathbf{x})+\theta_{g}^{T} \xi_{g}(\mathbf{x})
$$

If, instead, the variable of interest involved in transform (17) is the integral of tracking error, we obtain the so-called integral sliding mode control. Specifically, if transformation (17) is defined by

$$
s_{i}=\left(\frac{d}{d t}+\lambda\right)^{n} \varsigma(t)=\mathbf{c}^{T} \mathbf{e}(t)+c_{0} \zeta(t)
$$

where $\zeta(t)=\int_{0}^{t} e(\tau) d \tau$. Differentiation of $s_{i}$ with respect to $t$, yields

$$
\begin{aligned}
\dot{s}_{i} & =\mathbf{c}^{T} \dot{\mathbf{e}}+c_{0} e=\mathbf{c}^{T} \mathbf{A}_{c} \mathbf{e}+\mathbf{c}^{T} \mathbf{b}_{\mathbf{c}}\left(f(\mathbf{x})+g(\mathbf{x}) u-y_{d}^{(n)}\right)+c_{0} e \\
& =\overline{\mathbf{c}}^{T} \mathbf{e}+\left(f(\mathbf{x})+g(\mathbf{x}) u-y_{d}^{(n)}\right)+c_{0} e \\
& =f(\mathbf{x})-w_{i}+g(\mathbf{x}) u
\end{aligned}
$$

where $w_{i}=\overline{\mathbf{c}}_{i}^{T} \mathbf{e}+y_{d}^{(n)}=\sum_{i=0}^{n-1} c_{i} e^{(i)}+y_{d}^{(n)}$.

Similarly, the equivalent control input (19) is modified into

$$
\hat{u}_{e q}=-\frac{1}{\hat{g}(\mathbf{x})}\left[\hat{f}(\mathbf{x})-w_{i}\right]
$$

Next, we will define an additional complementary transformation to the generalized error transformation (22) such that a more meaningful error measure may be obtained. Corresponding to the same parameter $\lambda>0$, define

$$
s_{c}=\left(\frac{d}{d t}+\lambda\right)^{n-1}\left(\frac{d}{d t}-\lambda\right) \zeta(t)
$$

A striking result concerning about the relationship between $s_{i}$ and $s_{c}$ is

$$
\dot{s}_{c}+\lambda\left(s_{i}+s_{c}\right)=\dot{s}_{i}
$$

\subsection{Main results}

The control input is also composed of two terms, namely,

$$
u=\hat{u}_{e q}+u_{s}
$$

with $\hat{u}_{e q}$ defined in (24) and $u_{s}$ defined by

$$
u_{s}=-\frac{1}{\hat{g}(\mathbf{x})} K \operatorname{sgn}\left(s_{i}+s_{c}\right)
$$

It is easy to show the control input (27) will result in a closed-loop system satisfying the reaching condition

$$
\frac{1}{2} \frac{d}{d t} s^{2} \leq-\eta|s|
$$

Finally, from definitions (22) and (25) of $s_{i}$ and $s_{c}$, respectively, one has

$$
\frac{s_{i}+s_{c}}{2}=\left(\frac{d}{d t}+\lambda\right)^{n-1} e=s
$$

Substituting the control law given by (27) into (23) yields

$$
\begin{aligned}
\dot{s} & =f(\mathbf{x})-w+g(\mathbf{x}) u \\
& =f(\mathbf{x})-\hat{f}(\mathbf{x})+(g(\mathbf{x})-\hat{g}(\mathbf{x})) \hat{u}_{e q}+g(\mathbf{x}) u_{s}
\end{aligned}
$$

With the definition of (14) and (15), equation (31) can be expressed as

$$
\dot{s}=f^{*}(\mathbf{x})-\hat{f}(\mathbf{x})+\left(g^{*}(\mathbf{x})-\hat{g}(\mathbf{x})\right) \hat{u}_{e q}+g(\mathbf{x}) u_{s}+r
$$

where

$$
\mathrm{r}=f(\mathbf{x})-f^{*}(\mathbf{x})+\left(g(\mathbf{x})-g^{*}(\mathbf{x})\right) \hat{u}_{e q}
$$

is the reconstruction error.

From definition (20) and (21), respectively, one has

$$
\dot{s}=\Phi_{f}^{T} \xi_{f}(\mathbf{x})+\Phi_{g}^{T} \xi_{g}(\mathbf{x}) \hat{u}_{e q}+g(\mathbf{x}) u_{s}+r
$$

where $\Phi_{f}^{T}=\theta_{f}^{*}-\theta_{f}, \Phi_{g}^{T}=\theta_{g}^{*}-\theta_{g}$ and $\xi_{f}(\mathbf{x}), \quad \xi_{g}(\mathbf{x})$ are fuzzy basis functions.

The control input will be designed for compensating the variations of the unknown parameters such that the tracking error $e(t)$ will be driven arbitrarily close to zero. To this end, choose the following Lyapunov function candidate.

$$
\begin{aligned}
V & =\frac{1}{2}\left(\left(s_{i}+s_{c}\right)^{2}+\frac{1}{\gamma_{f}} \Phi_{f}^{T} \Phi_{f}+\frac{1}{\gamma_{g}} \Phi_{g}^{T} \Phi_{g}\right) \\
& =\frac{1}{2}\left(4 s^{2}+\frac{1}{\gamma_{f}} \Phi_{f}^{T} \Phi_{f}+\frac{1}{\gamma_{g}} \Phi_{g}^{T} \Phi_{g}\right)
\end{aligned}
$$

where $\gamma_{f}, \gamma_{g}$ are arbitrary positive numbers. The derivative of $V$ along the system trajectories is

$$
\begin{aligned}
\dot{V}= & 2 s^{2}-\frac{1}{\gamma_{f}} \Phi_{f}^{T} \dot{\vartheta}_{f}-\frac{1}{\gamma_{g}} \Phi_{g}^{T} \dot{\theta}_{g} \\
= & 2 s\left\{g(\mathbf{x}) u_{s}+r\right\}+\frac{1}{\gamma_{f}} \Phi_{f}^{T}\left(-\dot{\theta}_{f}+2 \gamma_{f} s \xi_{f}(\mathbf{x})\right) \\
& +\frac{1}{\gamma_{g}} \Phi_{g}^{T}\left(-\dot{\theta}_{g}+2 \gamma_{g} s \xi_{g}(\mathbf{x}) \hat{u}_{e q}\right)
\end{aligned}
$$

The adaptation laws for $\theta_{f}$ and $\theta_{g}$ are designed as follows:

$$
\begin{gathered}
\dot{\theta}_{f}=\gamma_{f}\left(s_{i}+s_{c}\right) \xi_{f}(\mathbf{x}) \\
\dot{\theta}_{g}=\gamma_{g}\left(s_{i}+s_{c}\right) \xi_{g}(\mathbf{x}) \hat{u}_{e q}
\end{gathered}
$$

Substituting the adaptation laws given by (37) and (38) into (36) equation (36) can be expressed as

$$
\begin{aligned}
\dot{V} & =2 s\left\{g(\mathbf{x}) u_{s}+r\right\} \\
& =2|s|\left\{-\frac{g(\mathbf{x})}{\hat{g}(\mathbf{x})} K+r \operatorname{sgn}(s)\right\}
\end{aligned}
$$

In order to satisfy $\dot{V}<0$, the switching gain $K$ is designed as follows

$$
K \geq \frac{\hat{g}^{U}}{g_{L}} r_{\max }
$$

where $g_{L}=\inf _{x \in U_{x}} g(\mathbf{x}), \hat{g}^{U}=\sup _{\theta_{q} \in \Omega_{q} x \in U_{x}} \hat{g}(\mathbf{x})$. 


\subsection{Control gain $K$}

In this section we present a solution to the sliding mode control design using concepts from fuzzy set theory. In the design of switching control $u_{s}$, the control gain $K$ has to be selected according to the following rules. At each instant time, $t$, $\left|s_{i}+s_{c}\right|$ is the algebraic value of the switching function $s$. Then these rules are:

$$
\begin{array}{ll}
\text { Rule 1: IF }\left|s_{i}+s_{c}\right| \text { is } S L & \text { THEN } K(t)=K_{L} \\
\text { Rule 2: IF }\left|s_{i}+s_{c}\right| \text { is } S M & \text { THEN } K(t)=K_{M} \\
\text { Rule 3: IF }\left|s_{i}+s_{c}\right| \text { is } S S & \text { THEN } K(t)=K_{S} \\
\text { Rule 4: IF }\left|s_{i}+s_{c}\right| \text { is } S Z & \text { THEN } K(t)=K_{Z}
\end{array}
$$

where $S L, S M, S S$ and $S Z$ are fuzzy sets of the variable $\left|s_{i}+s_{c}\right| \cdot K_{L}, K_{M}, K_{S}$ and $K_{Z}$ are different values of the control gain corresponding to a large, medium, small and zero gain value, respectively.

Given the value $\left|s_{i}+s_{c}\right|$ at the instant time, $t$, the value of the control gain $K$ at the time $t$ is inferred using the above four fuzzy if then rules following procedure.

$$
K(t)=\frac{\mu_{S L} K_{L}+\mu_{S M} K_{M}+\mu_{S S} K_{S}+\mu_{S Z} K_{Z}}{\mu_{S L}+\mu_{S M}+\mu_{S S}+\mu_{S Z}}
$$

This strategy of selecting the variable control gain $K(t)$ has following advantages over choosing a fixed control gain $K$ :

1) A large control gain is applied only when the system state is far away from the sliding mode.

2) When the system state is close to the sliding manifold, a small control gain used.

\subsection{Modification of the rules}

Control rules that are the most important factor in FLC are generally obtained from intuition and experience of the experts, and such rules represented by linguistic rule sets or fuzzy relation. But there is always something difficult to obtain from such control rules and this makes the design of the controller difficult. Here, a self-organizing fuzzy sliding mode control algorithm using gradient descent method is proposed.

The learning algorithm is to modify the consequent parameter such that the system trajectory stays on the sliding manifold. If the sliding condition, $s \dot{s}<0$ is, is satisfied, the switching function will converge to zero, leading to the desired dynamics. According to the sliding condition, the consequent parameters should be adjusted in the direction that minimizes the value $s \dot{s}$. These consequent parameters are adjusted to reduced $-s \dot{s}$ means that the controller is tuned to satisfy the sliding condition and consequently have a sliding behavior.

According to the gradient descent method, the parameters are updated from

$$
\dot{K}_{j}=-\Gamma \frac{\partial(-s \dot{s})}{\partial K_{j}(t)}
$$

in which $\Gamma$ is the learning gain. By the chain rule, (42) becomes

$$
\begin{aligned}
\dot{K}_{j} & =\Gamma \frac{\partial s \dot{s}}{\partial u(t)} \frac{\partial u(t)}{\partial K_{j}(t)} \\
& =\Gamma \frac{\partial s \dot{s}}{\partial \hat{u}_{e q}} \frac{\partial \hat{u}_{e q}}{\partial K_{j}}+\Gamma \frac{\partial s \dot{s}}{\partial u_{s}} \frac{\partial u_{s}}{\partial K_{j}}
\end{aligned}
$$

As $\hat{u}_{e q}$ depends on current state variable only, $\frac{\partial \hat{u}_{e q}}{\partial K_{j}}=0$.

Therefore we have

$$
\dot{K}_{j}=\Gamma \frac{\partial s \dot{s}}{\partial u_{s}} \frac{\partial u_{s}}{\partial K_{j}}
$$

Using (31) we can find

$$
\frac{\partial \dot{s} \dot{s}}{\partial u_{s}}=\operatorname{sg}(\mathbf{x})
$$

Also using (28) and (41) the switching control input is calculated as follows.

$$
u_{s}=-\frac{1}{\hat{g}(\mathbf{x})} \operatorname{sgn}(2 s) \frac{\sum_{j \in J} \mu_{j} K_{j}}{\sum_{j \in J} \mu_{j}}
$$

Substituting (45) and (46) into (44), we obtain the following learning law for consequent parameters:

$$
\dot{K}_{j}=-\Gamma \frac{g(\mathbf{x})}{\hat{g}(\mathbf{x})}|s| \frac{\mu_{j}}{\sum_{j \in J} \mu_{j}}
$$

Because the sign of $g(\mathbf{x})$ is generally known we approximate the learning law for consequent parameters as follows.

$$
\dot{K}_{j} \cong-\Gamma|s| \frac{\mu_{j}}{\sum_{j \in J} \mu_{j}}
$$

\section{Computer Simulations}

We apply the stabilization algorithm given in the previous section to balance an inverted pendulum mounted on a cart. The equations of motion for the pendulum are

$$
\begin{aligned}
& \dot{x}_{1}=x_{2} \\
& \dot{x}_{2}=\frac{g \sin \left(x_{1}\right)-a m l x_{2}^{2} \sin \left(2 x_{1}\right) / 2-a \cos \left(x_{1}\right) u}{4 l / 3-a m l \cos ^{2}\left(x_{1}\right)}
\end{aligned}
$$

where $x_{1}$ is the angle of the pendulum from the vertical line, $x_{2}$ is the angular velocity of the pendulum, $u$ is the control force applied to the cart, and $g=9.8 \mathrm{~m} / \mathrm{s}^{2}$ is the magnitude of the acceleration due to gravity.

Numerical values of the parameters are given in Table 1 .

The control objective is to track the reference signal $y_{d}=\frac{\pi}{30} \sin (t)$. The initial state is $\mathbf{x}(0)=\left[\begin{array}{ll}0.2 & 0\end{array}\right]^{T}$ and the sampling time is $1 \mathrm{~ms}$. In design example we choose the switching surface given by 


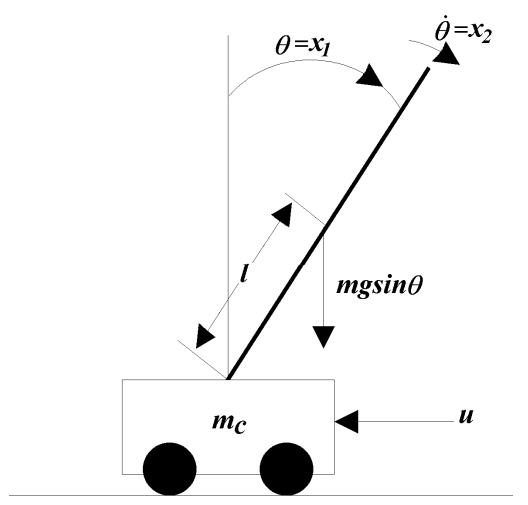

Fig.1 The inverted pendulum system

Table 1 The parameters of the inverted pendulum

\begin{tabular}{|c|c|c|}
\hline & symbol & Value \\
\hline Acceleration of gravity & $g$ & $9.8 \mathrm{~m} / \mathrm{s}^{2}$ \\
\hline Mass of cart & $m_{c}$ & $1 \mathrm{~kg}$ \\
\hline Mass of pole & $m$ & $0.5 \mathrm{~kg}$ \\
\hline Length of pole & $l$ & $0.5 \mathrm{~m}$ \\
\hline
\end{tabular}

$$
\begin{gathered}
s_{i}=\dot{e}+20 e+100 \int e d \tau=0 \\
s_{c}=\dot{e}-100 \int e d \tau=0 \\
\frac{s_{i}+s_{c}}{2}=\dot{e}+10 e=s
\end{gathered}
$$

The membership function for $x_{1}$ and $x_{2}$ are chosen as a Gaussian-shaped in Fig. 2.

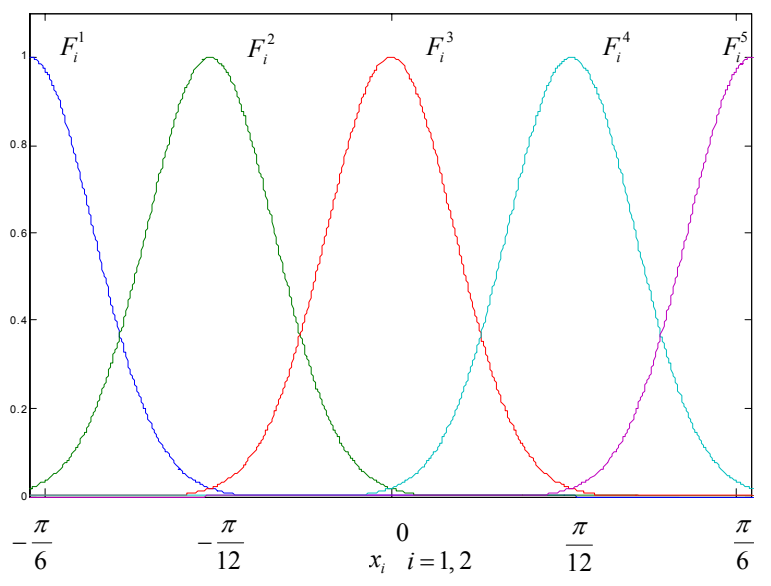

Fig.2 Fuzzy basis functions

For the proposed control algorithm, the membership functions of the fuzzy subsets SZ, SS, SM and SL are shown in Figure 3.

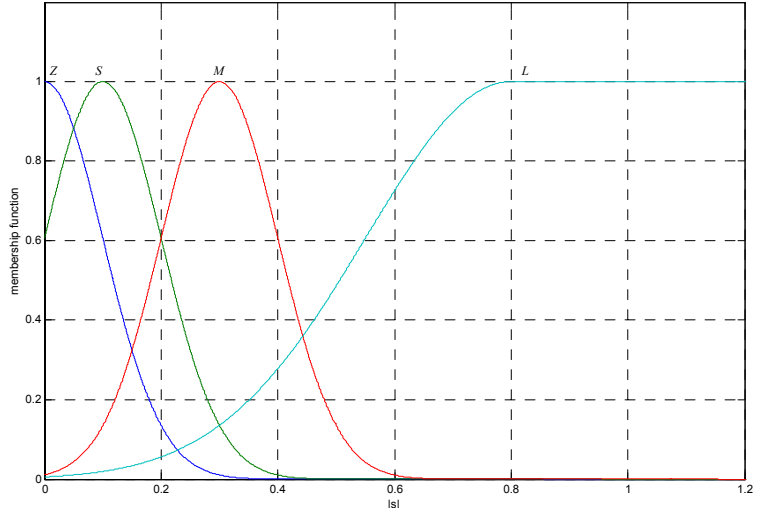

Fig.3 The membership functions of $\left|s_{i}+s_{c}\right|$

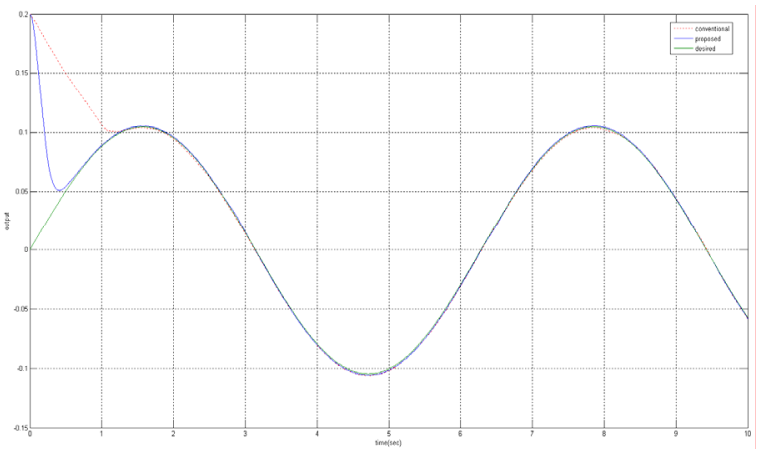

Fig.4 System output and desired trajectory

The simulation results are depicted in Figure 4 and Fig 5. By comparison, the tracking precision is significantly improved by the complementary control. The error trajectory starting from any initial state $(e=0.2, \dot{e}=0)$ approaches to the zero very quickly when the complementary sliding mode control was employed.

Fig 6. illustrates the history of control input $u$. It is clear from the simulation results that the proposed control approach reduces the chattering.

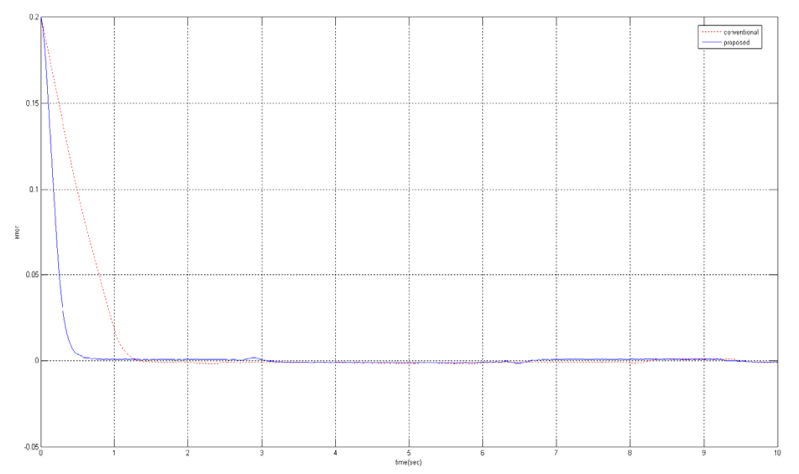

Fig.5 Trajectories of error 


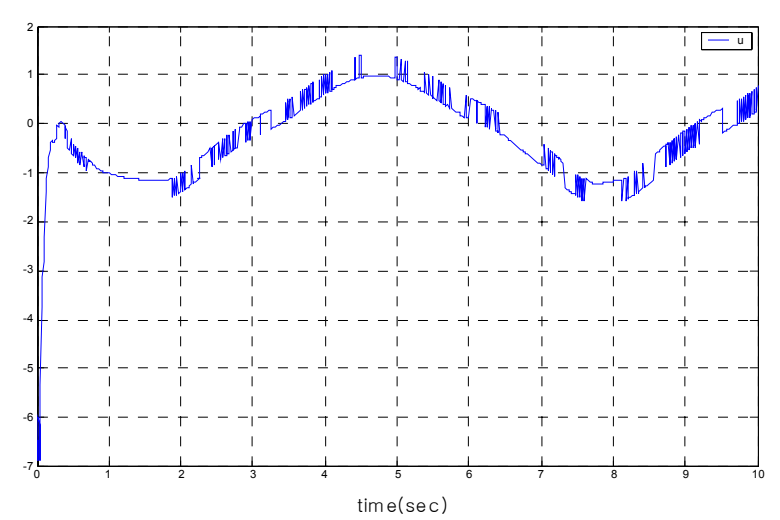

Fig.6 Control input

\section{Conclusions}

In this paper, an adaptive fuzzy sliding mode controller for a class of uncertain nonlinear dynamical systems is proposed and analyzed. The controller' $\mathrm{s}$ construction and its analysis involve sliding modes. The proposed controller consists of two components. Sliding mode component is employed to eliminate the effects of disturbances, while a fuzzy model component equipped with an adaptation mechanism reduces modeling uncertainties by approximating model uncertainties. To demonstrate its performance, the proposed control algorithm is applied to an inverted pendulum. The results show that both alleviation of chattering and performance are achieved.

\section{References}

[1] R. A. DeCarlo, S. H. Zak and G. P. Matthews, "Variable structure control of nonlinear multi variable systems : a tutorial", Proceedings of the IEEE, Vol. 76, No. 3, pp. 212232, 1988.

[2] K. K. D. Young, "Controller design of a manipulator using theory of variable structure systems", IEEE Trans. Syst. Man and Cybern., Vol. 8, No. 2, pp. 101-109, 1978.

[3] J. J. Slotine and S. S. Sastry, "Tracking control of nonlinear systems using sliding surfaces with applications to robot manipulators", Int. Journal of Control, Vol. 38, No. 2, pp. 465-492. 1983.
[4] L. A. Zadeh, "Fuzzy sets", Information and Control, Vol. 8, pp. 338-353, 1965.

[5] L. A. Zadeh, "Fuzzy algorithms", Information and Control, Vol. 12, pp. 94-102, 1968.

[6] D. Driankov, H. Hellendoorn and M. Reinfrank, An Introduction to Fuzzy Control, Springer-Verlag, NewYork, 1993.

[7] R. Palm, "Sliding mode fuzzy control", Proceedings of the IEEE International Conference on Fuzzy Systems, SanDiego, CA, pp. 519-526, 1992.

[8] R. Palm, "Robust control by fuzzy sliding mode", Automatica, Vol. 30, No. 9, pp. 1429-1437, 1994.

[9] U. Itkis, Control Systems of Variable Structure, John Wiley \& Sons, NewYork, 1976.

[10]M. W. Spong, and M. Vidyasager, Robot Dynamics and Control, John Wiley \& Sons, NewYork, 1989.

[11] S. H. Zak and J. J. Slotine, "Stabilizing Fuzzy System Models using Linear Controller", IEEE Trans. Fuzzy Systems, Vol. 7, No. 2, pp. 236-240, 1999.

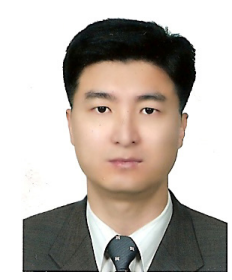

Sam-Jun Seo is received his B.S., M.S. and $\mathrm{Ph} . \mathrm{D}$. degrees in Electrical Engineering from Korea University in 1989, 1991 and 1996, respectively. He joined Anyang University in 1997 and is currently a professor in Electrical and Electronic Engineering. His research interests include intelligent control, fuzzy systems, neural networks, intelligent robot and tele-operating systems.

Phone : 031-467-0874

Fax : 031-467-0800

E-mail :ssj@anyang.ac.kr 